\title{
REDUCED NUMBER OF DESIGN PARAMETERS IN OPTIMUM PATH SYNTHESIS WITH TIMING OF FOUR-BAR LINKAGE
}

\author{
JACEK BUŚKIEWICZ \\ Poznan University of Technology, Institute of Applied Mechanics, Poznań, Poland \\ e-mail: jacek.buskiewicz@put.poznan.pl
}

\begin{abstract}
The paper presents the method for the optimal synthesis of four-link mechanism generating open/closed paths with time prescription. Although the method is suitable for both closed and open paths, it enables decreasing the number of design parameters describing dimensions, orientation and position of a path generator. Compared to the methods presented in the references, this is a one-phase synthesis method; although the number of design parameters is reduced, the method does not require affine transformations to be performed on the synthesised mechanism. The effectiveness of the method is discussed based on examples of three paths, with two taken from the literature.
\end{abstract}

Keywords: path synthesis, evolutionary algorithm, four-bar linkage

\section{Introduction}

Recent literature reveals a great variety of problems related to the path synthesis in which dimensions of a mechanism are sought, the point of which traces a desired path. This diversification may be classified with respect to:

1. Methods of solutions: analytical, computer (deterministic, heuristic).

2. Formulation of the problem: closed- or open-path synthesis, path synthesis with or without timing, one- or two-phase path synthesis.

3. Type of method: direct or indirect synthesis method (based on an atlas of coupler curves).

Owing to great mathematical complexity (Erdman et al., 2001), computer methods are mainly developed. The prevalent techniques are based on the genetic/evolutionary algorithms and other heuristic methods patterned upon biological systems and social behaviours (Avilés et al., 2010; Bulatović et al., 2013, 2016; Buśkiewicz, 2009, 2015; Buśkiewicz et al., 2010; Cabrera et al., 2007, 2011; Ebrahimi and Payvandy, 2015; Gogate and Matekar, 2012; Kafash and Nahvi, 2015; Kunjur and Krishnamurty, 1997; Lin, 2010; Matekar and Gogate, 2012; Nadal et al., 2015; Penunuri et al., 2011; Shiakolas et al. 2002, 2005). Frequently, the methods are devoted to a single specific path-synthesis task: synthesis of open-path generators, synthesis of closed-path generators, path synthesis with and without timing (time prescription). In the path synthesis with timing, each position of the coupler point corresponds to a prescribed angular position of the active link. In many cases, this correspondence is of less significance. In the open-path synthesis, a part of the coupler trajectory is prescribed.

The two-phase synthesis (Buśkiewicz et al., 2009; Buśkiewicz, 2010; Lin, 2014, 2015; McGarva and Mullineux, 1993; Nadal et al., 2015; Sanchez Marin and Gonzalez, 2004; Smaili and Diab, 2007; Sun et al., 2015; Ullah and Kota, 1997) for the direct synthesis method consists of two steps. At first the shape synthesis is carried out followed by scale-rotation-translation transformations. The first step reduces the number of design variables. This number is important when a synthesis method is based on deterministic-probabilistic algorithms for optimal parameter determination. 
Every additional parameter increases the dimension of the solution space and may increase the computational cost. In general, the effectiveness of a method depends essentially on the number of design parameters, by which the objective function is expressed.

This paper deals with the direct synthesis method dedicated for an open/closed-path synthesis with timing. The mathematical foundations of the usage of the Fourier descriptors (FDs) as shape signatures (descriptors) of a closed curve were formulated by Zahn and Roskies (1972). Although a prevailing number of these methods may be used for closed paths, the wavelet transform has been used to describe open paths by Sun et al. (2015). Smaili and Diab (2007) presented the two-phase synthesis method based on the so-called cyclic angular deviation vector. Buśkiewicz et al. (2009) proposed a two-phase synthesis method in which the shape was described by means of the curvature-based Fourier descriptors. The centroid, the direction of the major principal axis and the perimeter of a curve enabled translation, rotation and scaling of the mechanism to the desired configuration. Buśkiewicz (2010) also proposed a two-phase synthesis method using the function of the distance of the curve from its centroid, to describe the curve shape in terms of its normalised Fourier coefficients. Sanchez Marin and Gonzalez (2004) utilised geometric properties to describe the path (without addressing harmonic series theories) and to reduce the number of optimised parameters to five in the open-path synthesis.

The methods using decomposition of the path into normalised parameters, invariant with respect to affine transformation, also belongs to the so-called indirect synthesis method (Chu and Sun, 2010; Galán-Marín et al., 2009; Hoeltzel and Chieng, 1990; McGarva, 1994; Mullineux, 2011; Sun and Chu, 2009; Vasiliu and Yannou, 2001; Yu et al., 2007, 2012). The second phase consists of searching through a computerised atlas database of normalised descriptors which are linked to dimensions of the mechanisms generating closed paths. Nonetheless, most techniques are onephase syntheses which either introduce new error functions or develop algorithms evaluating an objective function (Acharyya and Mandal, 2009; Avilés et al., 2010; Bulatović and Dordević, 2009; Bulatović et al., 2013, 2016; Cabrera et al., 2002, 2007, 2011; Ebrahimi and Payvandy, 2015; Gogate and Matekar, 2012; Khorshidi et al., 2011; Kinzel et al., 2006; Kunjur and Krishnamurty, 1997; Lio 1997; Lio et al., 2000; Lin, 2010; Lin and Hsiao, 2017; Matekar and Gogate, 2012; Penunuri et al., 2011, Sancibrian et al., 2004; Schmiedeler et al., 2014; Shiakolas et al., 2002, 2005; Smaili et al., 2005). The number of design parameters is as large as necessary to define the problem. In the optimum synthesis with timing the design variables are not only mechanism dimensions and orientation but also angular positions of the input link corresponding with the prescribed coupler points.

The number of techniques for open/closed-path synthesis with timing, defined by the decreased number of design parameters, is rather small (Nadal et al., 2015; Kafash and Nahvi, 2015). The paper presents the method for the optimal synthesis of a four-link planar mechanism generating open paths with time prescription, enabling a decrease in the number of design parameters describing dimensions, orientation and position of the path generator. Compared with the methods presented in the references, this is a one-phase synthesis method; i.e. although the number of design parameters is reduced, the method does not require affine transformations to be performed on the synthesised mechanism. The paper is a continuation of the work (Buśkiewicz, 2015) dealing with the one-phase path synthesis without timing, defined by means of a reduced number of design parameters.

\section{Method description}

\subsection{Mathematical foundations}

The method for path synthesis with timing is aimed at minimising the number of design variables being optimised using a heuristic algorithm. The coupler curve is defined by means of the discrete number of points. When the input link rotates by a given angle, the coupler point 
passes between two neighbouring curve points. The geometric scheme of the four-bar linkage is shown in Fig. 1a.

The design variables optimised by means of the algorithm are (Fig. 1b):

- coordinates of the input link pivot: $x_{O 1}, y_{O 1}$,

- input link length: $l_{1}$, and coupler $A B$ length: $l_{2}$,

- angle between arms $A D$ and $A B$ of the coupler: $\theta_{4}$,

- angular position of the input link $\theta_{10}$ corresponding with the first coupler point.

Six parameters define the four-bar linkage instead of the 10 required in the classical approach to path synthesis with timing $\left(l_{1}, l_{2}, l_{3}, l_{4}, l 5, x_{O 1}, y_{O 1}, \theta_{4}, \gamma, \theta_{10}\right)$.

The input data are given as set $\left\{\left(x_{i}, y_{i}, \theta_{1 i}\right), i=1, \ldots, n\right\}$, where $\left(x_{i}, y_{i}\right)$ are the coordinates of the points to be drawn by the coupler point $D$ for the input link angular position $\theta_{1 i}^{\prime}=\theta_{1 i}+\theta_{10}$. It means that passing from the point $i$ to the point $i+1$ is accompanied by rotation of the input link by the angle $\theta_{1 i+1}-\theta_{1 i}$. The angle $\gamma$ is determined in the phase of the computations and is not known in the initial phase. This is why the angular position of the input link $\theta_{1}$ is measured from the horizontal line. Let us assume that we have a set of arbitrary values of the design variables defining a candidate for the optimum mechanism. One has to evaluate how accurately this mechanism approximates the given performance.

(a)

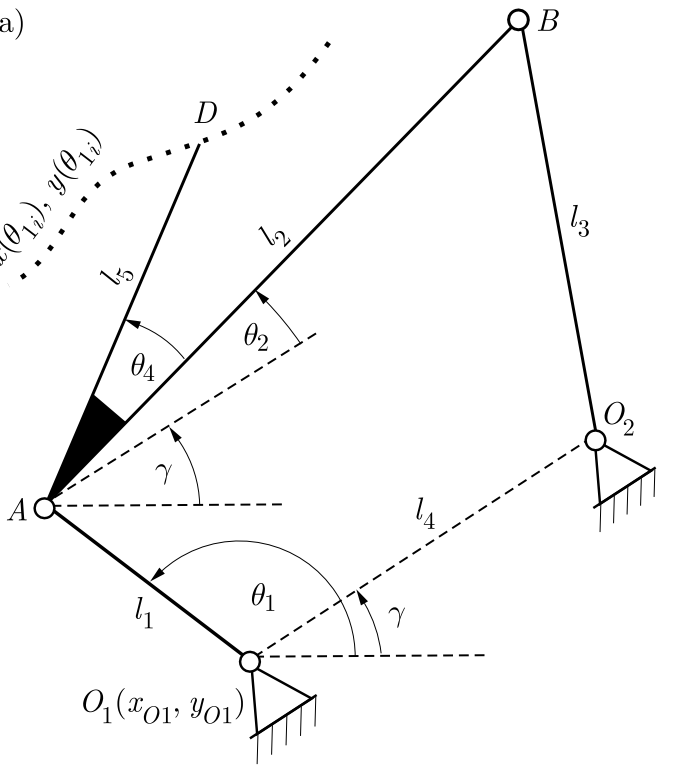

(b)

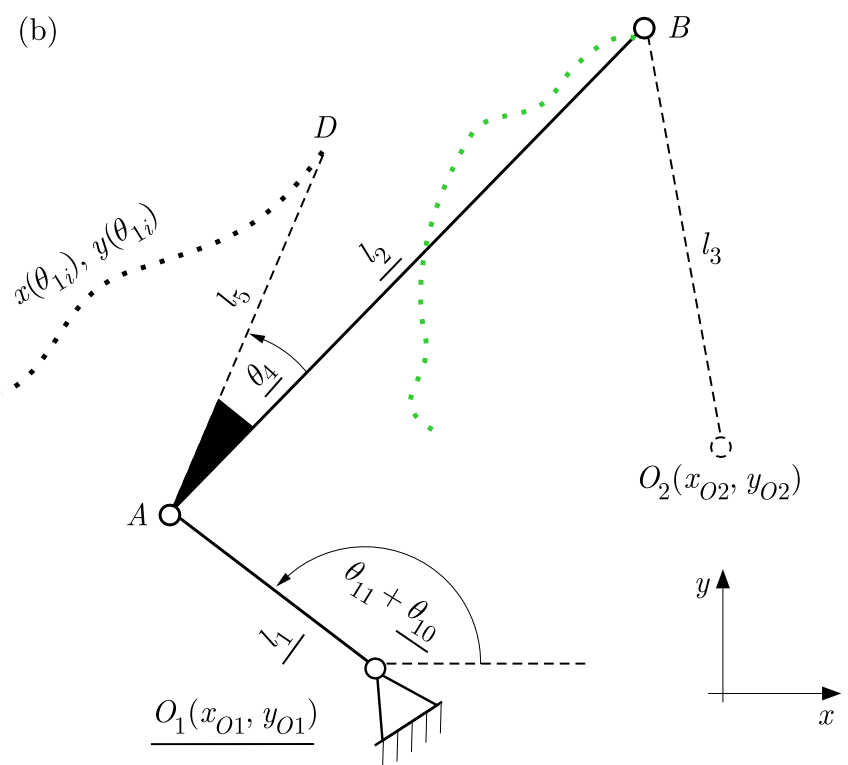

Fig. 1. (a) General geometric scheme of the four-bar linkage, (b) design variables (underlined) and variables determined using design variables

The algorithm for determination of the remaining dimensions of the mechanism is as follows.

Having certain design variables $x_{O 1}, y_{O 1}, l_{1}$ and $\theta_{10}$, the positions of the joint $A$ on the input link for $i=1, \ldots, n$ are computed

$$
x_{A i}=x_{O 1}+l_{1} \cos \left(\theta_{1 i}+\theta_{10}\right) \quad y_{A i}=y_{O 1}+l_{1} \sin \left(\theta_{1 i}+\theta_{10}\right)
$$

Subsequently, the lengths are computed

$$
\left|A_{i} D_{i}\right|=\sqrt{\left(x_{A i}-x_{i}\right)^{2}+\left(y_{A i}-y_{i}\right)^{2}}
$$

as well as the trigonometric functions between the section $A_{i} D_{i}$ and the horizontal line

$$
\cos \alpha_{i}=\frac{x_{i}-x_{A i}}{\left|A_{i} D_{i}\right|} \quad \sin \alpha_{i}=\frac{y_{i}-y_{A i}}{\left|A_{i} D_{i}\right|}
$$


The optimum length of the arm $|A D|$ minimises, in the sense of the least square method, the sum of the squared deviations of the prescribed point $D$ positions from the points generated by dyad $O_{1} A D$, defined by $x_{O 1}, y_{O 1}, l_{1}, \theta_{10}$ and $l_{5}$ for the prescribed angular positions of the link $O_{1} A$

$$
E_{1}=\sum_{i=1}^{n}\left[\left(x_{A i}+l_{5} \cos \alpha_{i}-x_{i}\right)^{2}+\left(y_{A i}+l_{5} \sin \alpha_{i}-y_{i}\right)^{2}\right]
$$

As $l_{5}$ is not known, differentiating $E_{1}$

$$
\frac{\partial E_{1}}{\partial l_{5}}=2 \sum_{i=1}^{n}\left[\left(x_{A i}+l_{5} \cos \alpha_{i}-x_{i}\right) \cos \alpha_{i}+\left(y_{A i}+l_{5} \sin \alpha_{i}-y_{i}\right) \sin \alpha_{i}\right]=0
$$

one can obtain

$$
l_{5}=\frac{1}{n} \sum_{i=1}^{n}\left[\left(x_{A i}-x_{i}\right) \cos \alpha_{i}+\left(y_{A i}-y_{i}\right) \sin \alpha_{i}\right]
$$

which equals the average value of $\left|A_{i} D_{i}\right|$ for all $i$.

Then the desired design variables minimise the deviations of $\left|A_{i} D_{i}\right|$ from $l_{5}$. The next set of design variables are the angle between the coupler arms $A D$ and $A B-\theta_{4}$ and the coupler $A B$ length $-l_{2}$. Having these values, one can compute the position of the joint $B$ connecting the coupler and the output link. Using the complex number notation, we have the subsequent positions of the link $\mathrm{AB}$ corresponding with the positions of the link $A D$

$$
\begin{gathered}
\mathbf{A}_{i} \mathbf{B}_{i}=\left(x_{B i}-x_{A i}\right)+\mathrm{j}\left(y_{B i}-y_{A i}\right)=l_{2} \frac{\mathbf{A}_{i} \mathbf{D}_{i}}{\left|A_{i} D_{i}\right|} \mathrm{e}^{\mathrm{j}\left(-\theta_{4}\right)} \\
=l_{2}\left(\cos \alpha_{i}+\mathrm{j} \sin \alpha_{i}\right)\left[\cos \left(-\theta_{4}\right)+\mathrm{j} \sin \left(-\theta_{4}\right)\right]
\end{gathered}
$$

Hence, the coordinates

$$
x_{B i}=x_{A i}+l_{2} \cos \left(\alpha_{i}-\theta_{4}\right) \quad y_{B i}=y_{A i}+l_{2} \sin \left(\alpha_{i}-\theta_{4}\right)
$$

are obtained by clockwise rotating the unit vector $\mathbf{A}_{i} \mathbf{D}_{i}$ by the angle $\theta_{4}$ and multiplicating the result by $l_{2}$. It is expected that the position of the joint $B$ can be approximated by a circular arc. The radius of the arc that best fits into these points equals the length of the output link $O_{2} B$, and the centre of the arc becomes the pivot of this link. The parameters of this circular arc may be computed using the method described in (Buśkiewicz, 2015). Let $l_{3 i}$ denote the radius of the circle with the centre at $\left(x_{O_{2} i}, y_{O_{2} i}\right)$ passing through three points: $B_{i}, B_{i^{\prime}}$ and $B_{i^{\prime \prime}}$, where: $i=1, \ldots, n, i^{\prime}=i+2, i^{\prime \prime}=i^{\prime}+2$, and the number point greater than $n$ is diminished by $n$. The centre coordinates of the $i$-th circle are

$$
x_{O_{2} i}=\frac{b_{1} a_{22}-b_{2} a_{12}}{a_{11} a_{22}-a_{12} a_{21}} \quad y_{O_{2} i}=\frac{b_{2} a_{11}-b_{1} a_{21}}{a_{11} a_{22}-a_{12} a_{21}}
$$

where

$$
\begin{aligned}
& a_{1}=2\left(x_{B i}-x_{B i^{\prime}}\right) \quad a_{12}=2\left(y_{B i}-y_{B i^{\prime}}\right) \\
& a_{21}=2\left(x_{B i}-x_{B i^{\prime \prime}}\right) \quad a_{22}=2\left(y_{B i}-y_{B i^{\prime \prime}}\right) \\
& b_{1}=x_{B i}^{2}+y_{B i}^{2}-x_{B i^{\prime}}^{2}-y_{B i^{\prime}}^{2} \quad b_{2}=x_{B i}^{2}+y_{B i}^{2}-x_{B i^{\prime \prime}}^{2}-y_{B i^{\prime \prime}}^{2}
\end{aligned}
$$

Then, the coordinates of the ground pin are

$$
x_{O_{2}}=\frac{1}{n} \sum_{i=1}^{n} x_{O_{2} i} \quad y_{O_{2}}=\frac{1}{n} \sum_{i=1}^{n} y_{O_{2} i}
$$


Similarly, the average radius is taken as the length of the output link

$$
l_{3}=\frac{1}{n} \sum_{i=1}^{n} l_{3 i}=\frac{1}{n} \sum_{i=1}^{n}\left|O_{2} B_{i}\right|
$$

The final step is to compute the length of the immovable link, and cosine and sine of the angle $\gamma$

$$
l_{4}=\left|O_{1} O_{2}\right| \quad \cos \gamma=\frac{x_{O 2}-x_{O 1}}{l_{4}} \quad \sin \gamma=\frac{y_{O 2}-y_{O 1}}{l_{4}}
$$

An alternative method for measuring the similarity of the set of points to the circular curve has recently been presented by Kafash and Nahvi (2015). The Circular Proximity Function is defined as

$$
C P F=\sum_{i=1}^{n}\left[\left(x_{B i}-x_{O 2}\right)^{2}+\left(y_{B i}-y_{O 2}\right)^{2}-R^{2}\right]
$$

where the average value of the squared distances between the points $B$ and the centre of the hypothetical circle is as follows:

$$
R=\frac{1}{n} \sum_{i=1}^{n}\left[\left(x_{B i}-x_{O 2}\right)^{2}+\left(y_{B i}-y_{O 2}\right)^{2}\right]
$$

The optimum circle centre coordinates minimises the $C P F$.

\subsection{Objective function and Euclidian error}

The four-bar linkage realises exactly the desired motion when the sum of deviations of the arm length $l_{5}$ from real distances $\left|A_{i} D_{i}\right|$ and deviations of the length of the output link from computed real distances $\left|O_{2} B_{i}\right|$ (the measure of the deviation of the path of the joint $B$ from an ideal circular arc)

$$
\delta=\frac{\max \left|A_{i} D_{i}\right|-\min \left|A_{i} D_{i}\right|}{l_{5}}+\frac{\max \left|O_{2} B_{i}\right|-\min \left|O_{2} B_{i}\right|}{l_{3}}
$$

equals 0 . Then $\delta$ is the minimised objective function and, simultaneously, it is the indirect measure of the inaccuracy of the method. This error bears no information about the Euclidian deviation between given points and points traced by the mechanism obtained. For the determined dimensions of the four-bar linkage, the coordinates of the coupler points for the given angular positions of the input link are computed. For this purpose, the angular position of the coupler and the output link $\mathrm{O}_{2} B$

$$
\theta_{21,2}=2 \arctan \frac{-K_{E} \pm \sqrt{K_{E}^{2}-4 K_{D} K_{F}}}{2 K_{D}} \quad \theta_{31,2}=2 \arctan \frac{-K_{B} \pm \sqrt{K_{B}^{2}-4 K_{A} K_{C}}}{2 K_{A}}
$$

are determined, where

$$
\begin{aligned}
& K_{A}=K_{3}-K_{1}+\left(1-K_{2}\right) \cos \left(\theta_{1}-\gamma\right) \quad K_{B}=-2 \sin \left(\theta_{1}-\gamma\right) \\
& K_{C}=K_{1}+K_{3}-\left(1+K_{2}\right) \cos \left(\theta_{1}-\gamma\right) \quad K_{D}=K_{5}-K_{1}+\left(1+K_{4}\right) \cos \left(\theta_{1}-\gamma\right) \\
& K_{E}=-2 \sin \left(\theta_{1}-\gamma\right) \quad K_{F}=K_{1}+K_{5}+\left(K_{4}-1\right) \cos \left(\theta_{1}-\gamma\right) \quad K_{1}=\frac{l_{4}}{l_{1}} \\
& K_{2}=\frac{l_{4}}{l_{3}} \quad K_{3}=\frac{l_{1}^{2}-l_{2}^{2}+l_{3}^{2}+l_{4}^{2}}{2 l_{1} l_{3}} \quad K_{4}=\frac{l_{4}}{l_{2}} \quad K_{5}=\frac{-l_{1}^{2}-l_{2}^{2}+l_{3}^{2}-l_{4}^{2}}{2 l_{1} l_{2}}
\end{aligned}
$$


Each solution corresponds to the two configurations of the four-bar linkage in which the mechanism can be assembled for the pre-set dimensions and the angular position of link $O_{1} A$. At this stage, the mechanism whose configuration has been generated is not known, therefore the errors for both signs at the square roots in Eqs. (2.16) are computed and the more accurate solution is taken into account.

Then the position of the point $D$ at the instant when the angular position of the input link measured from the horizontal line equals to $\theta_{1 i}+\theta_{10}$ is determined from the equations

$$
\begin{aligned}
& x_{D i}=x_{O 1}+l_{1} \cos \left(\theta_{1 i}+\theta_{10}\right)+l_{5} \cos \left[\theta_{2}\left(\theta_{1 i}+\theta_{10}-\gamma\right)+\theta_{4}+\gamma\right] \\
& y_{D i}=y_{O 1}+l_{1} \sin \left(\theta_{1 i}+\theta_{10}\right)+l_{5} \sin \left[\theta_{2}\left(\theta_{1 i}+\theta_{10}-\gamma\right)+\theta_{4}+\gamma\right]
\end{aligned}
$$

The angular position of the crank with respect to the axis $O_{1} O_{2}$ equals $\theta_{1 i}+\theta_{10}-\gamma$. Therefore, this angle is taken to compute $\theta_{2}$ from Eq. (2.16). In the references, the absolute Euclidian error between the points given and generated is defined as follows

$$
E=\sum_{i=1}^{n}\left[\left(x_{D i}-x_{i}\right)^{2}+\left(y_{D i}-y_{i}\right)^{2}\right]
$$

\subsection{Optimisation technique}

The evolutionary algorithm (EA) (Goldberg, 1989) minimises the objective function (Eq. (2.15)). The technique is described in depth in (Buśkiewicz, 2010, 2015). Preliminary numerical simulations were performed to establish the parameters controlling the EA:

- The size of the population $N_{\max }=80$ (case I), $N_{\max }=50$ (cases II and III).

- The number of individuals to be crossed over in each generation $l_{c r}=30$.

- The number of randomly generated individuals introduced to each population in the place of the worst fitted ones $l_{r n d}=25$.

- A feature of the new individual $(\mathrm{N})$ is inherited from the parent (I) with the probability $p_{b}=0.75$ (from parent (II) with the probability 0.25 ).

- The initial value of the mutation coefficient $\lambda=0.05$ (the value is gradually decreased to 0.005 ), the initial disturbance coefficient $\mu=0.001$ is gradually decreased to 0.00001 ).

- The minimum value of the error function (objective function) $E_{\min }$ depends on the case being solved.

- The maximum number of iterations executed (generations) in the case of not achieving the prescribed minimum value of the error function $L_{g}=10000$.

\section{Numerical solutions}

To prove the effectiveness of the method, three examples are solved and discussed. Two of them are taken from references and comparative analysis is carried out.

For all cases presented the points are gathered in sets in the forms as follows: $t a b=$ $\left\{\left(x_{i}, y_{i}, \theta_{1 i}\right), i=1, \ldots, n\right\}$. The coordinates are non-dimensional, the angles are in radians.

\section{Case 1}

The first case was presented by Acharyya and Mandal (2009). The authors of this paper considered the synthesis with timing defined by data given in $t a b_{1}$

$$
\begin{aligned}
& t a b_{1}=\{(0,0,0.5236),(1.9098,5.878,1.0472),(6.9098,9.511,1.5708),(13.09,9.511,2.0944), \\
& \quad(18.09,5.878,2.618),(20,0,3.1416)\}
\end{aligned}
$$


The input geometric constraints were:

- the range of the coordinates of the crank pivot: $-50 \leqslant x_{O 1}, y_{O 1} \leqslant 50$,

- the lengths of the crank: $5 \leqslant l_{1} \leqslant 50$, and of the coupler: $5 \leqslant l_{2} \leqslant 50$.

The computational time was 00:01:14 with 1000 iterations (generations in EA - 80000 evaluations of the objective function) performed. The evaluations of chosen parameters are shown in Fig. 2a. The progress of the absolute Euclidian error is presented in Fig. 2b.
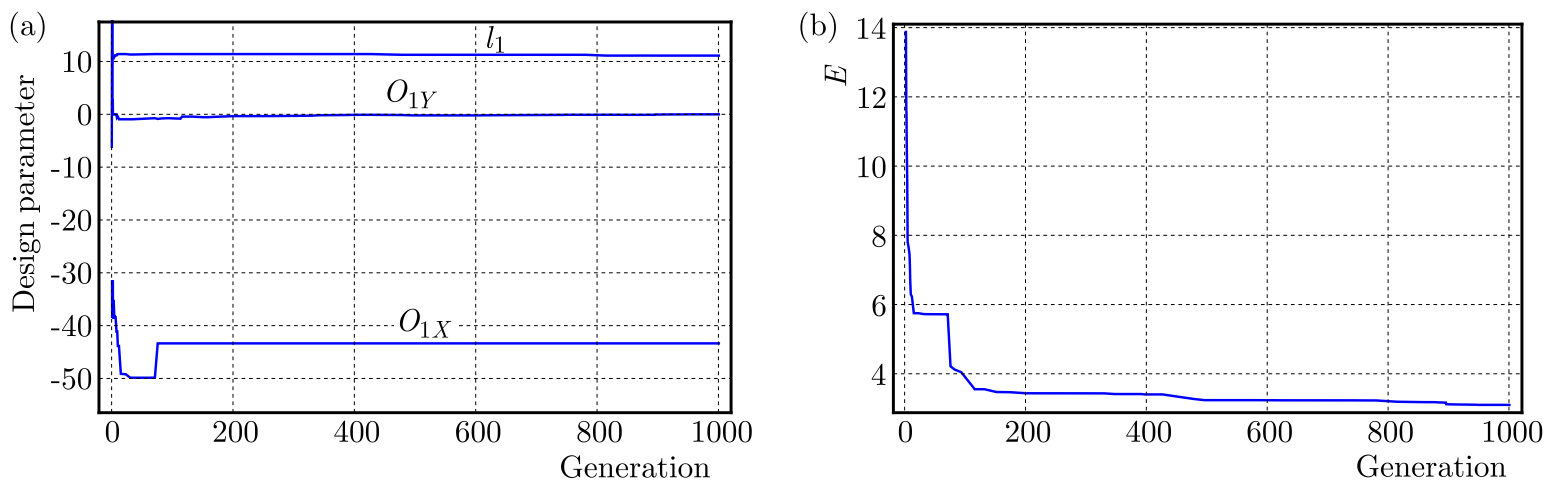

Fig. 2. (a) Evaluation of the crank length and crank pivot coordinates. (b) Evaluation of the Euclidian distance

It is visible from Figs. 2a,b that the areas surrounding the found values of parameters are localised in the first 200 iterations. The best solution of the problem with installing parameters for the first position (Fig. 3) is: $l_{1}=11.1149, l_{2}=42.6226, \theta_{4}=$ $-0.1368 \mathrm{rad}, \gamma=6.2819 \mathrm{rad}, \theta_{10}=3.29 \mathrm{rad}, l_{3}=11.9381, l_{5}=52.4112, l_{4}=43.30492$, $O_{1}(-43.3598,-0.0067), O_{2}(-0.0549,-0.0609), D(-0.143478,0.271792), A(-52.058,-6.92637)$, $B(-11.032,4.63172)$ and $\theta_{1}=\theta_{10}+\theta_{11}\left(=t a b_{11,3}\right)=3.8136 \mathrm{rad}$ (the angle is measured form the horizontal line).

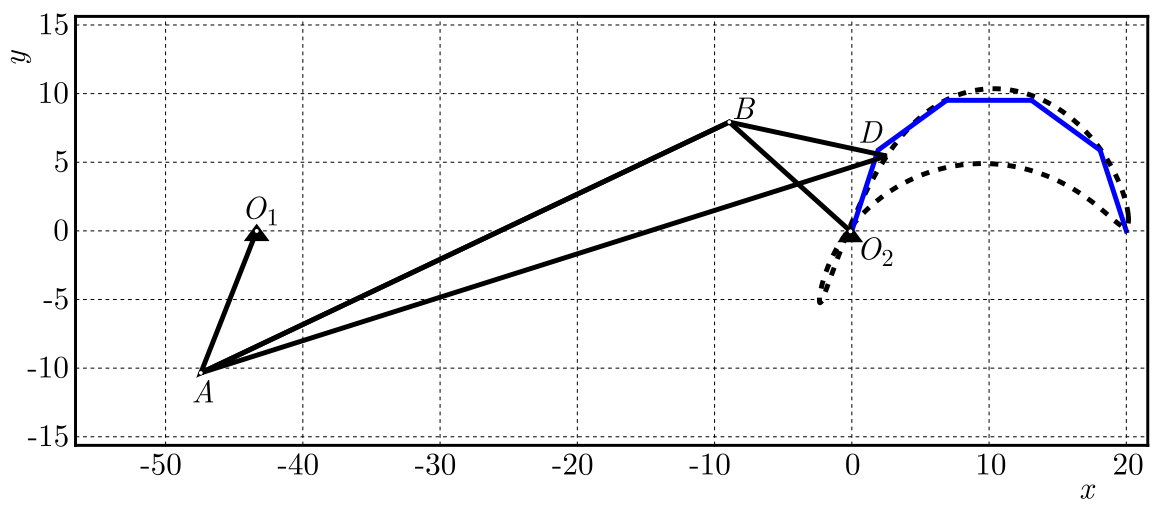

Fig. 3. Case 1: Synthesised four-bar linkage with paths given (continuous line) and generated

The method error $\delta=2.767$, and the absolute Euclidian error $E=2.10037$. Three EAs namely Genetic Algorithm (GA), Particle Swarm Optimization (PSO) and Differential Evolution (DE) were applied separately in (Acharyya and Mandal, 2009). The best solution obtained using DE is presented in Table 1. The error reported by the authors is 2.349649 , whereas the error computed for the corresponding mechanism dimensions is 5.52074 - this difference was also observed by Ebrahimi and Payvandy (2015). In all addressed methods 100000 or 200000 evaluations of the objective function were performed, whereas in the presented paper there were 80000 . The four-bar linkages generating this path were also determined in (Ebrahimi and Payvandy, 2015) using: imperialist competitive algorithm (ICA) and parallel simulated annealing 
(SA). The best solution is also presented in Table 1. Ebrahimi and Payvandy also solved the problem of the path synthesis with workspace limits, which is not here considered. The ICA and EA differ from each other in terms of the structure and, therefore, it is difficult to compare their computational costs of the evaluations of the objective functions.

Table 1. Path 1: comparison of results

\begin{tabular}{|c|c|c|c|}
\hline & $\begin{array}{c}\text { Acharyya and } \\
\text { Mandal (2009) } \\
\text { DE }\end{array}$ & $\begin{array}{c}\text { Ebrahimi and } \\
\text { Payvandy (2015) } \\
\text { ICA }\end{array}$ & Proposed \\
\hline \hline$l_{1}$ & 5 & 5 & 11.1149 \\
\hline$l_{2}$ & 5.905345 & 7.08248 & 42.6226 \\
\hline$l_{3}$ & 50 & 48.05733 & 11.9381 \\
\hline$l_{4}$ & 50 & 50 & 43.30492 \\
\hline$l_{5}$ & 18.819312 & 21.3969 & 52.4112 \\
\hline$\theta_{4}$ & 0 & 0.6956 & -0.1368 \\
\hline$x_{O 1}$ & 14.373772 & 11.88034 & -43.3598 \\
\hline$y_{01}$ & -12.444295 & -16.08766 & -0.0067 \\
\hline$\gamma$ & 0.463633 & 6.2831853 & 6.2819 \\
\hline$\theta_{10}$ & - & - & 3.29 \\
\hline$E$ & 5.52074 & 2.5998 & 2.10037 \\
\hline
\end{tabular}

\section{Case 2}

Kunjur and Krishnamurty (1997) presented the synthesis with timing of the path given by 18 points:

$$
\begin{aligned}
& t a b_{2}=\{(0.5,1.1,0.3491),(0.4,1.1,0.6981),(0.3,1.1,1.0472),(0.2,1.0,1.3963), \\
& (0.1,0.9,1.7453),(0.05,0.75,2.0944),(0.02,0.6,2.4435),(0,0.5,2.7925),(0,0.4,3.1416), \\
& (0.03,0.3,3.4907),(0.1,0.25,3.8397),(0.15,0.2,4.1888),(0.2,0.3,4.5379), \\
& \quad(0.3,0.4,4.8869),(0.4,0.5,5.2360),(0.5,0.7,5.5851),(0.6,0.9,5.9341),(0.6,1,6.2832)\}
\end{aligned}
$$

The range for the coordinates of the crank pivot is: $-50 \leqslant x_{O 1}, y_{O 1} \leqslant 50$. The lengths of the crank and coupler $l_{1}, l_{2}$ cannot exceed 50. The computational time is 00:00:36. Within this time, 200 iterations have been performed - the number of evaluations of the objective function is 10000 . The optimum solution is expressed as follows: $l_{1}=0.4102, l_{2}=1.2166$, $\theta_{4}=0.8342853 \mathrm{rad}, \gamma=0.1110 \mathrm{rad}, l_{3}=1.1230, l_{5}=0.7859, l_{4}=1.5395, \theta_{10}=0.8721 \mathrm{rad}$.

These parameters are completed with installing parameters for the first position (Fig. 4): $O_{1}(0.363,-0.0874), O_{2}(1.8931,0.0831), D(0.516114,1.08379), A(0.503501,0.297987)$, $B(1.41786,1.10058), \theta_{1}=\theta_{10}+\theta_{11}\left(=t a b_{21,3}\right)=1.2212 \mathrm{rad}$.

The absolute Euclidian error $E=0.0185453$, and the method error $\delta=0.1821$. Taking into account the solutions obtained by means of the EAs (Kunjur and Krishnamurty, 1997; Cabrera et al. 2011; Nadal et al. 2015), the method presented in this paper gives the best solution. The number of evaluations of the objective functions in these methods is 5000. In order to provide higher repeatability, when 34 in 100 algorithm executions give the solution with error $E<0.02$, twice as many evaluations of the objective function in the presented method are taken. In order to obtain more accurate solutions, Lin and Hsiao (2017) employed the Cuckoo Search (CS) and teaching-learning-based optimization (TLBO) algorithms (200 001 evaluations of the objective function - twice as many evaluations resulted only in the smaller values of the mean error 


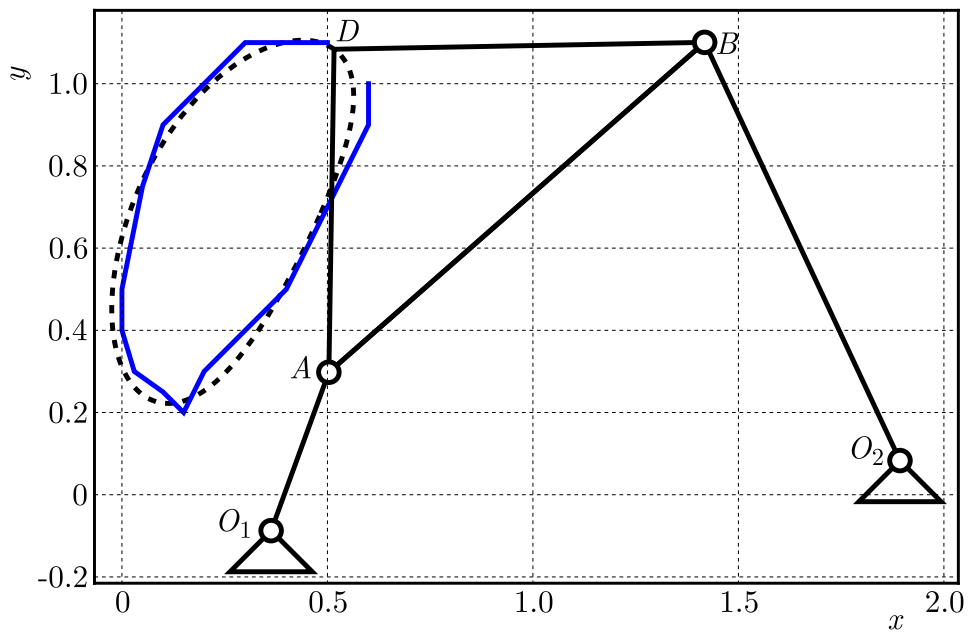

Fig. 4. Case 2: Synthesised four-bar linkage with paths given (continuous line) and generated

and standard deviation). The Modified Krill Herd algorithm applied by Bulatović et al. (2016) produced the same degree of accuracy. The number of the objective function evaluations in the $\mathrm{MKH}$ algorithm is the product of the number of iterations and the number of repetitions - both parameters are equal to 50. Compared with the EAs, the computational cost of one evaluation of the objective function seems to be higher. This issue, however, was not discussed in (Bulatović et al., 2016) since the MKH, as was the case with the ICA, differred from the EA in performance. All the results are summarised in Table 2.

Table 2. Path 2: comparison of the results

\begin{tabular}{|c|c|c|c|c|c|c|}
\hline & $\begin{array}{c}\text { Kunjur and } \\
\text { Krishnamurty } \\
(1997)\end{array}$ & $\begin{array}{c}\text { Cabrera } \\
\text { et al. } \\
(2011)\end{array}$ & $\begin{array}{c}\text { Nadal } \\
\text { et al. } \\
(2015)\end{array}$ & $\begin{array}{c}\text { Bulatović } \\
\text { et al. } \\
(2016)\end{array}$ & $\begin{array}{c}\text { Lin and } \\
\text { Hsiao } \\
(2017)\end{array}$ & Proposed \\
\hline \hline$l_{1}$ & 0.274853 & 0.297057 & 0.239834 & 0.4218 & 0.4238752 & 0.4102 \\
\hline$l_{2}$ & 1.180253 & 3.913095 & 0.941660 & 0.87821 & 0.9142488 & 1.2166 \\
\hline$l_{3}$ & 2.138209 & 0.849372 & 3.164512 & 0.58013 & 0.5989170 & 1.1230 \\
\hline$l_{4}$ & 1.879660 & 4.453772 & 2.462696 & 1.00429 & 1.0539434 & 1.5395 \\
\hline$l_{5}$ & 0.91561 & 2.65198 & 0.4834 & 0.5234 & 0.54481 & 0.7859 \\
\hline$\theta_{4}$ & 3.5680854 & 2.464734 & 1.5638233 & 0.8147729 & 0.822721 & 0.8342853 \\
\hline$x_{O 1}$ & 1.132062 & -1.309243 & 0.569026 & 0.26886 & 0.2676546 & 0.363 \\
\hline$y_{O 1}$ & 0.663433 & 2.806964 & 0.350557 & 0.17715 & 0.1546514 & -0.0874 \\
\hline$\gamma$ & 4.354224 & 2.7387359 & 4.788536 & 0.29294 & 0.2848225 & 0.1110 \\
\hline$\theta_{10}$ & 2.558625 & 4.853543 & 2.47266 & 0.88595 & 0.8915568 & 0.8721 \\
\hline$E$ & 0.043 & 0.0196 & 0.113595 & 0.00911 & 0.0090289 & 0.0185453 \\
\hline
\end{tabular}

\section{Case 3}

The set $t a b_{3}$ presents the straight line discretised by 6 points with the crank positions assigned to

$$
\begin{gathered}
t a b_{3}=\{(5.0000,1.000,0.3491),(4.0000,1.000,0.6981),(3.0000,1.000,1.0472), \\
(2.0000,1.000,1.3963),(1.0000,1.000,1.7453),(0.0000,1.000,2.0944)\}
\end{gathered}
$$

The input geometric constraints define the range for the crank pivot coordinates: $-25 \leqslant x_{O 1}$, $y_{O 1} \leqslant 25$, and the maximum permissible lengths of the crank and coupler equal to 15 . The 
computational time is 00:00:47 with 1000 iterations performed for $N_{\max }=50$. The geometric parameters of the optimum mechanism are as follows: $l_{1}=1.7930, l_{2}=6.6176, \theta_{4}=0.4732 \mathrm{rad}$, $\gamma=2.6679 \mathrm{rad}, \theta_{10}=4.5633 \mathrm{rad}, l_{3}=6.6175, l_{5}=11.7914, l_{4}=3.475$.

The absolute Euclidian error $E=0.0000173$, and method error $\delta=0.0038$. Taking into account the absolute error, one can state that the solution is very accurate. The installing parameters for the first position (Fig. 5) are: $O_{1}(5.5219,-9.0021), O_{2}(2.4294,-7.4167), D(4.99683,0.99906)$, $A(5.87813,-10.7594), B(8.44532,-4.65988) . \theta_{1}=\theta_{10}+\theta_{11}\left(=t a b_{31,3}\right)=4.9124 \mathrm{rad}$.

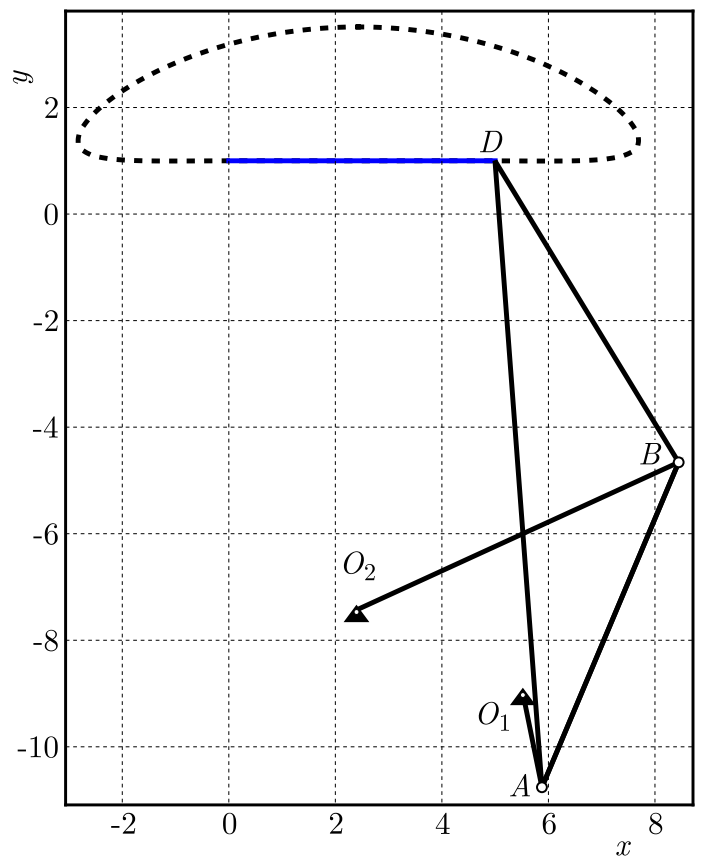

Fig. 5. Case 3: Synthesised four-bar linkage with paths given (continuous line) and generated

\section{Conclusion}

As mentioned in the introduction, two main branches have been developed recently in the field of mechanism synthesis. The first one deals with the enhancement of probabilistic deterministic algorithms. The other focuses on the minimisation of the number of design variables. It is difficult to find papers on the reduction of the number of design parameters in the case of open/closed-path synthesis with timing in the one-phase path synthesis. The presented method tries to overcome these difficulties and this is the distinctive feature of the method. Free paths are considered to investigate the effectiveness of the method. Although the algorithm employed in this paper utilizes the classical EA, the results of the synthesis are comparable to the best of those presented in the literature. The analysis performed is encouraging for further studies in developing the methods decreasing the number of design parameters, which may be an alternative to more complex, frequently hybrid, new optimisation algorithms. The method, although presented on the example of a four-bar linkage, can be applied in synthesis of other four-link planar mechanisms: crank-slider mechanism and mechanism with slotted links using techniques described in (Buśkiewicz, 2015).

One can formulate some general observations on the way the results obtained by many authors are compared. First of all, it seems reasonable to take into account some measure which reflects the ratio of sizes of the mechanism and the synthesised path. Frequently, the working space occupied by the optimal mechanism when tracing the path is much larger than the space enclosed by the path alone. Such a solution is frequently useless for practical implementation. 
Moreover, in the case of an open-path synthesis, the general tendency is that a better solution is characterised by a smaller ratio of the length of the synthesised part to the total path length. This means that the path is generated for a very small angle of rotation of the input link, and the greater part of the mechanism motion is dead motion. Therefore, the comparison of different methods based on the values of the objective functions should only be concluded very carefully.

Such an approach to mechanism synthesis, which consists of reducing the number of design parameters, imposes distinction between the problems with and without timing. In other methods, when the problem without timing is considered, the orientations of the input link corresponding with the coupler point synthesis are treated as additional design parameters.

Acknowledgment

This research was funded by Polish Ministry of Science and Higher Education (the grant DS 02/21/DSPB/3493).

\section{References}

1. Acharyya S.K., Mandal M., 2009, Performance of EAs for four-bar linkage synthesis, Mechanism and Machine Theory, 44, 9, 1784-1794

2. Avilés R., Vallejo J., Fernández de Bustos I., Aguirrebeitia J., Ajuria G., 2010, Optimum synthesis of planar linkages using a strain-energy error function under geometric constraints, Mechanism and Machine Theory, 45, 65-79

3. Bulatović R.R., Dordević S.R., 2009, On the optimum synthesis of a four-bar linkage using differential evolution and method of variable controlled deviations, Mechanism and Machine Theory, 44, 235-246

4. Bulatović R.R., Dordević S.R., Dordević V.S., 2013, Cuckoo Search algorithm: A metaheuristic approach to solving the problem of optimum synthesis of a six-bar double dwell linkage, Mechanism and Machine Theory, 61, 1-13

5. Bulatović R.R., Miodragović G., Bošković M.S., 2016, Modified Krill Herd (MKH) algorithm and its application in dimensional synthesis of a four-bar linkage, Mechanism and Machine Theory, 95, 1-21

6. BuŚKIEWICZ J., 2010, Use of shape invariants in optimal synthesis of geared five-bar linkage, Mechanism and Machine Theory, 45, 273-290

7. BuśKiEwiCZ J., 2015, A method for optimal path synthesis of four-link planar mechanisms, Inverse Problems in Science and Engineering, 23, 818-850

8. Buśkiewicz J., Starosta R., Walczak T., 2009, On the application of the curve curvature in path synthesis, Mechanism and Machine Theory, 44, 1223-1239

9. Cabrera J.A., Nadal F., Munoz J.P., Simon A., 2007, Multiobjective constrained optimal synthesis of planar mechanisms using a new evolutionary algorithm, Mechanism and Machine Theory, 42, 791-806

10. Cabrera J.A., Ortiz A., Nadal F., Castillo J..J., 2011, An evolutionary algorithm for path synthesis of mechanisms, Mechanism and Machine Theory, 46, 127-141

11. Cabrera J.A., Simon A., Prado M., 2002, Optimal synthesis of mechanisms with genetic algorithms, Mechanism and Machine Theory, 37, 10, 1165-1175

12. Chu J., Sun J., 2010, Numerical atlas method for path generation of spherical four-bar mechanism, Mechanism and Machine Theory, 45, 6, 867-879

13. Ebrahimi S., Payvandy P., 2015, Efficient constrained synthesis of path generating four-bar mechanisms based on the heuristic optimization algorithms, Mechanism and Machine Theory, 85, 189-204 
14. Erdman A.G., Sandor G.N., Kota S.S., 2001, Mechanism Design: Analysis and Synthesis, 4th Ed. (Web Enhanced), Volume I, Prentice-Hall

15. Galán-Marín G.F., Alonso J., Del Castillo J.M., 2009, Shape optimization for path synthesis of crank-rocker mechanisms using a wavelet-based neural network, Mechanism and Machine Theory, 44, 6, 1132-1143

16. Gogate G.R., Matekar S.B., 2012, Optimum synthesis of motion generating four-bar mechanisms using alternate error functions, Mechanism and Machine Theory, 54, 41-61

17. GoldBerg D.E., 1989, Genetic Algorithms in Search, Optimization and Machine Learning, Addison Wesley, Massachusetts

18. Hoeltzel D.A., Chieng W.H., 1990, Pattern matching synthesis as an automated approach to mechanical design, Journal of Mechanical Design, 112, 2, 190-199

19. Kafash S.H., Nahvi A., 2015, Optimal synthesis of four-bar motion generator linkages using circular proximity function, Proceedings of the Institution of Mechanical Engineers Part C Journal of Mechanical Engineering Science, 203-210, 1989-1996

20. Khorshidi M., Soheilypour M., Peyro M., Atai A., Panahi M.S., 2011, Optimal design of four-bar mechanisms using a hybrid multi-objective GA with adaptive local search, Mechanism and Machine Theory, 46, 10, 1453-1465

21. Kinzel E.C., Schmiedeler J.P., Pennock G.R., 2006, Kinematic synthesis for finitely separated positions using geometric constraint programming, Journal of Mechanical Design, 128, 5, 1070-1079

22. Kunjur A., Krishnamurty S., 1997, Genetic algorithms in mechanical synthesis, Journal of Applied Mechanisms and Robotics, 4, 2, 18-24

23. LiN W.Y., 2010, A GA-DE hybrid evolutionary algorithm for path synthesis of four-bar linkage, Mechanism and Machine Theory, 45, 8, 1096-1107

24. LiN W.Y., 2014, Optimization of scale-rotation-translation synthesis after shape synthesis for path generation of planar mechanisms, Journal of the Chinese Institute of Engineers, 37, 4, 497-505

25. LiN W.Y., 2015, Optimum synthesis of planar mechanisms for path generation based on a combined discrete Fourier descriptor, Journal of Mechanisms and Robotics, 7, 4, 041023-041023

26. Lin W.Y., Hsiao K.M., 2017, Cuckoo search and teaching-learning-based optimization algorithms for optimum synthesis of path-generating four-bar mechanisms, Journal of the Chinese Institute of Engineers, 40, 1, 66-74

27. Lio M.D., 1997, Robust synthesis of linkages-synthesis by solving non-linear optimization problem, Mechanism and Machine Theory, 32, 8, 921-932

28. Lio M.D., Cossalter V., Lot R., 2000, On the use of natural coordinates in optimal synthesis of mechanisms, Mechanism and Machine Theory, 35 ,10, 1367-1389

29. Matekar S.B., Gogate G.R., 2012, Optimum synthesis of path generating four-bar mechanisms using differential evolution and a modified error function, Mechanism and Machine Theory, 52, 158-179

30. McGarva J., Mullineux G., 1993, Harmonic representation of closed curves, Applied Mathematical Modelling, 17, 4, 213-218

31. MCGARVA J.R., 1994, Rapid search and selection of path generating mechanisms from a library, Mechanism and Machine Theory, 29, 2, 223-235

32. Mullineux G., 2011, Atlas of spherical four-bar mechanisms, Mechanism and Machine Theory, 46, 11, 1811-1823

33. Nadal F., Cabrera J.A., Bataller A., Castillo J.J., Ortiz A., 2015, Turning functions in optimal synthesis of mechanisms, Journal of Mechanical Design, 137, 6, 062302 (10 pages) 
34. Penunuri F., Peón-Escalante R., Villanueva C., Pech-Oy D., 2011, Synthesis of mechanisms for single and hybrid tasks using differential evolution, Mechanism and Machine Theory, 46, $10,1335-1349$

35. Sanchez Marin F.T., Gonzalez. A.P., 2004, Open-path synthesis of linkages through geometrical adaptation, Mechanism and Machine Theory, 39, 943-955

36. Sancibrian R., Viadero F., García P., Fernández A., 2004, Gradient-based optimization of path synthesis problems in planar mechanisms, Mechanism and Machine Theory, 39, 8, 839-856

37. Schmiedeler J.P., Clark B.C., Kinzel E.C., Pennock G.R., 2014, Kinematic synthesis for infinitesimally and multiply separated positions using geometric constraint programming, Journal of Mechanical Design, 136, 3, 034503 (7 pages)

38. Shiakolas P.S., Koladiya D., Kebrle J., 2002, On the optimum synthesis of four-bar linkages using differential evolution and the geometric centroid of precision positions, Inverse Problems in Science and Engineering, 10, 6, 485-502

39. Shiakolas P.S., Koladiya D., Kebrle J., 2005, On the optimum synthesis of six-bar linkages using differential evolution and the geometric centroid of precision positions technique, Mechanism and Machine Theory, 40, 319-335

40. Smaili A., Diab N., 2007, A new approach to shape optimization for closed-path synthesis of planar mechanisms, Journal of Mechanical Design, 129, 9, 941-948

41. Smaili A.A., Diab N.A., Atallah N.A., 2005, Optimum synthesis of mechanisms using TabuGradient search Algorithm, Journal of Mechanical Design, 127, 917-923

42. Sun J., Liu W., Chu J., 2015, Dimensional synthesis of open-path generator of four-bar mechanisms using the haar wavelet, Journal of Mechanical Design, 137, 8, 082303 (8 pages)

43. Sun J.W., Chu J.K., 2009, Fourier method to function synthesis of an RCCC mechanism, Proceedings of the Institution of Mechanical Engineers, Part C: Journal of Mechanical Engineering Science, 223, 2, 503-513

44. Ullah I., Kota S., 1997, Optimal synthesis of mechanisms for path generation using Fourier descriptors and global search methods, Journal of Mechanical Design, 119, 504-510

45. VAsiliu A., Yannou B., 2001, Dimensional synthesis of planar mechanisms using neural networks: application to path generator linkage, Mechanism and Machine Theory, 36, 2, 299-310

46. Yu H., TANG D., WAng Z., 2007, Study on a new computer path synthesis method of a four-bar linkage, Mechanism and Machine Theory, 42, 4, 383-392

47. Yue C., Su H.J., GE Q.J., 2012, A hybrid computer-aided linkage design system for tracing open and closed planar curves, Computer-Aided Design, 44, 11, 1141-1150

48. ZAhn T., Roskies R.Z., 1972, Fourier descriptors for plane closed curves, IEEE Transactions on Computers, 21, 3, 269-281 